\title{
How Can We Make Desalination More Reliable, Efficient and Sustainable?
}

\author{
Nikolay Voutchkov* \\ Water Globe Consultants, USA
}

*Corresponding author: Nikolay Voutchkov, Water Globe Consultants, LLC, USA.

\section{Introduction}

Utility leaders worldwide face multiple challenges to secure a reliable, efficient and sustainable future water supply - from accelerated population growth, to exhaustion of our traditional water sources, water scarcity driven by climate change and inefficient management of our available water resources. Finding a rational solution for sustainable water supply compels us to seek new water management solutions based on:

- $\quad$ Adopting the wider use of alternative water resources such as desalination of sea and brackish water

- Driving down the barriers for low energy desalination

- Extracting valuable resources (such as minerals and rare metals) from the waste streams generated during the water treatment processes, and

- Increasing reliance on renewable energy sources.

\section{How Do we Make Desalination More Sustainable?}

Desalination can provide a valuable tool to achieve water supply sustainability in the highly urbanized coastal areas of the world. Its raw material (the ocean) is practically limitless rendering desalination drought proof. In an uncertain world, desalination can significantly reduce climate change risks. The stable, efficient supply of urban and industrial water that desalination provides can help governments manage a range of economic, social, and political risks. The alarming increase in water scarcity across the world has led to the exponential growth of desalination as a drought-proof alternative to conventional surface water resources such as lakes, rivers and dams. Over the past 20 years the annual growth rate of new desalinated fresh water production capacity worldwide has been 4 to $8 \%$, several times more than the 1 to $2 \%$ increase in construction of new conventional water treatment plants.
However, local and regional sociopolitical dynamics are factors that may affect the viability of desalination as an alternative water supply in coastal areas. Experience has shown that the response to public opposition to new desalination plants in some parts of the world, such as California and Australia, is costly and timeconsuming, even preventing the development of new plants. The interplay of these parameters, whether environmental, economic, or sociopolitical should be taken into account when the strategic decision is made to consider desalination as a pathway to augmenting and increasing reliability of water supply.

Globally, the majority of desalination systems use reverse osmosis membrane separation technology due to its simplicity, efficiency and affordability. The rapid progress in membrane technology and treatment processes has made desalination more reliable and has supplied drought-proof water to many large urban coastal centers worldwide. There are currently over 20,000 desalination plants operating in 120 countries across the Middle East, North Africa, Australia, China and India among others. Innovative project designs and flexible funding schemes have driven the costs of desalination down to less than a dollar per cubic meter. However, today the desalination industry still faces the challenge of how to make it more affordable, sustainable and environmentally friendly.

\section{The key solutions to this challenge are:}

- Disruptive changes in desalination technology that yield significantly lower water production costs

- Further reduction of energy use and resultant carbon footprint of desalination by introducing a new generation of membranes, pump and energy recovery technologies 
- Wider use of technologies for zero and near-zero liquid discharge combined with beneficial use of the minerals and rear elements in the brine, and

- Adopting renewable sources of energy for desalination.

\section{Making Desalination More Affordable by Adopting New Disruptive Technologies}

Today reverse osmosis is a well-established and mature technology. But it has its limitations in terms of energy use and cost of key desalination plant equipment and membranes. Further reduction of these costs can be made possible by:

- Creating a new generation of nanostructured membranes of significantly higher productivity and ability to adjust membrane performance in-situ and on demand

- Extending the oxidation and biofouling resistance of membranes and their useful life

- $\quad$ Replacing most of the desalination plant steel equipment and piping with lower cost/lower weight plastic equipment and piping

- $\quad$ Onsite 3-D printing instead of shipping of spare parts for key equipment

- $\quad$ Adopting 3-D design, 3-D 0\&M manuals and 3-D virtual reality training for plant staff

- Use of low-cost 3-D printable wireless sensors for performance monitoring of all key plant components

- $\quad$ Automating plant monitoring and control to a level that allows remote unmanned plant operation, and

- Using separation technologies other than reverse osmosis.

\section{Breaking Down the Energy Barriers of Desalination}

The energy efficiency of desalination technologies has increased significantly over the past 30 years and energy use for production has been reduced to less than half. At present, the annual amount of energy used for production of desalinated water for a family of four is lower than the energy consumed by the family's refrigerator! Considering the importance of producing fresh drinking water for humanity at large, we as an industry are proud of our achievements to date in driving down the energy used for production of desalinated water.

We also have to keep in mind that the annual carbon footprint of desalinated water production ( 0.1 tons of carbon dioxide) is only $2.5 \%$ of the carbon footprint of the worldwide average of one person of 4 tons of carbon dioxide. In comparison, the average carbon footprint of hamburgers consumed by one person per year is four times higher at 0.4 tons of carbon dioxide per year. So, if everyone eats one less hamburger every year, we will be able to fully mitigate the carbon footprint of the desalinated water we drink!

Latest reverse osmosis technologies and designs of large desalination plants have yielded specific energy demand of less than $3.5 \mathrm{kWh}$ per cubic meter of fresh water produced. However, there is potential to further reduce energy demand to less than 2 kWh per cubic meter. The three barriers that currently drive the energy use of desalination are:

- The salinity of the source seawater

- Efficiency of the high-pressure pumps and energy recovery devices, and

- Energy losses through the membrane system.

\section{Can we reduce the salinity of source seawater?}

The latest trend in siting desalination plants with open intakes is to select the intake location at the point of exit of freshwater aquifer into the ocean, which usually yields 20 to $30 \%$ lower salinity than open ocean waters. This lower source water salinity yields proportionally lower energy use. An added bonus is the low algae content of this saline source water as seawater algae have low tolerance for low salinity water.

Another emerging trend is joint desalination and reuse. This method capitalizes on the lower salinity brine from advanced wastewater treatment plants (or brackish water desalination), mixed with seawater prior to entering the seawater desalination plant thereby decreasing the salinity of the feed water, and associated energy use. Such joint treatment has benefits beyond lower energy use which include higher plant recovery, freshwater production and reduced salinity of the plant discharge.

\section{How do we make pumps and energy recovery devices more efficient?}

At present, the high-pressure pumps for seawater reverse osmosis desalination plants consume over $70 \%$ of the total plant energy and have maximum efficiency of between 84 and $88 \%$, depending on the size of the pump. The efficiency of these pumps is limited by their type - centrifugal pump efficiency is inherently limited to approximately $90 \%$.

However, the $90 \%$ efficiency barrier can be overcome by the use of piston pumps, which have maximum efficiency of 99\%. To date, the use of piston pumps has not been widely adopted in membrane desalination plants due to their high pulsations which may damage reverse osmosis membranes.

Can we solve the high pulsation challenge of piston pumps? Yes - it has in fact already been solved by pressure exchanger energy recovery technology, which employs the piston pump principle for pump water with energy efficiency of over $96 \%$. The next step is to create a high-pressure piston pump that applies a similar pulsation attenuation solution as the one used in pressure exchanger energy recovery devices. So it is not a matter of whether it is possible - it is just a matter of time!

Since 2001, when the first pressure exchangers were introduced, this disruptive technology has increased the energy recovery efficiency in seawater desalination plants from 75 to $96 \%$. However, there is still an opportunity for the energy recovery barrier to be pushed closer to the theoretical maximum of $99 \%$. 


\section{How do we make desalination more environmentally friendly?}

Innovative project designs and flexible funding schemes have driven the cost of desalination down to less than a dollar per cubic meter. However, the environmental impacts of desalination provide many opportunities for improvement. Examples of such impacts include the effect of concentrate discharge on marine life, and impingement and entrainment of sea life in intake systems.

\section{So how do we make desalination more environmentally friendly?}

- $\quad$ By being better stewards of the environment

- By embracing the principles of the circular economy in brine management and looking at brine as a resource rather than waste, and

- $\quad$ By using alternative sources of energy for desalination different from fossil fuels.

\section{Being better stewards to the environment is of critical importance to the future of desalination}

The desalination industry is often challenged on the impact of brine discharges into the marine environment. In early 2019, an article was published by Bloomberg News, amongst others vilifying desalination discharges as toxic effluent and challenging the ability of the desalination industry to minimize the impact of such discharges on the environment. However, the reality is that today, desalination industry and regulators have a comprehensive system to predict, monitor and control the potential environmental impacts during all phases of project development and implementation from planning to design, construction and operation.

Desalination plant operators continuously monitor and comply with the strict environment regulations and discharge water quality standards set by pertinent local government agencies. During desalination project inception and planning phases, all contemporary desalination projects undergo a thorough Environmental Impact Assessment (EIA). Compliance with environmental impact mitigation measures delineated in the EIA is monitored and enforced by our construction management and regulatory compliance oversight teams throughout the project construction and commissioning process. This is also a condition for certification for completion of project construction to our contractors and approval of plant operations license and discharge permit.

The discharge permits of today's desalination plants contain comprehensive end-of-pipe water quality monitoring requirements and mandate periodic offshore marine environment monitoring studies to ascertain permit compliance and to identify and mitigate any potential environmental impacts. Besides environmental compliance monitoring professionals located at each desalination plant, desalination plants typically have an on-going water qualitymonitoring program. The state-of-the-art desalination processes employed in the desalination plants built over the last two decades use a very limited amount of chemicals. All chemicals added to enhance the quality of the produced water are of food grade, biodegradable, and specifically selected not to cause any aquatic marine life toxicity.

In addition, over the past several years, many utilities worldwide have initiated the implementation of a comprehensive program for green desalination, which has resulted in a further reduction of both the amount and types of chemicals used in the production of desalinated water. The green desalination programs ultimately aim to convert desalination facilities into chemicalplants by implementing the latest advancements of desalination technology and science.

Large desalination utilities, such as the Saline Water Corporation of Saudi Arabia which produces over 5.6 million cubic meters of desalinated water per day, have recently initiated comprehensive zero-liquid discharge programs that target significant reduction of the volume of concentrate discharges to the sea and to harvest minerals and rare earth metals from the desalination plant brine.

\section{Embracing circular economy by extracting valuable resources from brine}

Nature teaches us that sustainable existence of closed systems such as our planet has to rely on an efficient circular path when using resources like energy and water. A circular economy is the only path forward towards worldwide sustainable economic growth. Water utilities have the responsibility to transform water from a single-use resource to a precious, renewable commodity, and to incorporate this commodity into a robust circular economy. For example, applying the circular economy model, brine generated from desalination plants can be used as a source of valuable minerals, such as calcium, magnesium and sodium chloride. We can also extract rare-earth elements from brine including lithium, strontium and uranium.

Recent stresses in the global market of rare-earth elements have brought the availability and supply of technology metals to the forefront of the sustainability debate and research agenda. Metals are used to fabricate critical components of numerous products and finished goods, including airplanes, automobiles, smart phones, and biomedical devices. There is a growing realization that the development and deployment of clean energy technologies and sustainable products, processes and manufacturing industries of the 21st century will also require large amounts of critical metals and valuable elements including platinum group metals, lithium, copper, cobalt, silver, and gold.

The latest technology trends show that magnesium is replacing aluminum in the car, computer and cell phone industries because it is over $30 \%$ lighter. While the world's mining sources of magnesium are fairly limited, seawater brine contains very large quantities of magnesium which could be recovered by concentration of nanofiltration brine followed by selective extraction by adsorption. The desalination industry is making major strides towards beneficial reuse of concentrate. Recently developed technologies 
allow to generate two high mineral content streams from seawater treated sequentially by nanofiltration and reverse osmosis. The brine from nanofiltration membrane separation system is rich in calcium and magnesium and has low sodium chloride content. The brine from a downstream reverse osmosis system which is of very high sodium chloride content can be used directly as a source material by the chloralkaline industry.

In addition, brine can be used to add calcium and magnesium to the desalinated drinking water to re-mineralize it and to irrigate commercially valuable crops and fruits such as mangoes, after mixing it with treated wastewater. As a result, minerals in the brine are harvested and used beneficially for re-mineralization of the desalinated water as well as for raw materials used in chemical and high-tech industries, rather than returned back to the ocean.

Brine concentration and beneficial reuse have very positive impacts on the environment, resulting in zero or near-zero liquid discharge of brine into surface water bodies. Extracting minerals from seawater is also a more environmentally friendly enterprise than terrestrial mining. Moreover, seawater extraction will not require fresh water for processing nor create volumes of contaminated water or tailings for disposal.

This example shows that transitioning to a circular economy can achieve far more than reducing the negative impacts of the linear economy. Rather, it represents a systemic shift that builds longterm resilience, generates business and economic opportunities, and provides environmental and societal benefits.

\section{Use of alternative energy sources for desalination}

The use of alternative energy sources for desalination of seawater could provide the much-needed paradigm shift towards long-term sustainability of future water supply. The coupling of renewable energy with desalination has understandably attracted significant attention from the desalination industry worldwide. In 2018, the International Desalination Association's Global Clean Water Desalination Alliance has established a goal for at least 20 percent of the new plants to be powered by renewables between 2020-2025. To date there have been a number of small and medium solar desalination power projects in UAE and Saudi Arabia, combining concentrated solar power and seawater desalination. The challenge has been to scale the size of operations and make it fully independent, without access to grid power as backup when the sun isn't shining. To date practical experience shows the simplest configuration to be photovoltaic technology alongside that of reverse osmosis, and utilizing the grid for night supply, or as storage.
Currently the engineering firm Metito is working under contract with the King Abdullah Economic City (KAEC) to construct a seawater desalination plant powered by solar energy, valued at US $\$ 58.7$ million. The plant will initially have a capacity of 30,000 $\mathrm{m} 3$ of drinking water per day, expandable to $60,000 \mathrm{~m} 3$ per day. The project is expected to be in in production by the end of this year. Hoping to move the needle of desalination energy efficiency, UAE's Masdar implemented a Renewable Energy Water Desalination Program, which also advanced the full-scale implementation of renewable power-driven desalination technologies. While their work has shown that the most cost-effective approach for use of renewable energy currently is to decouple the desalination plant from the solar power generation system, the quest for solar power desalination is in a full swing. The decoupling is due to challenges with the quality of power and power storage and availability. However, further advancements of solar power battery storage technology hold high potential for continuous 24-hour solar energy driven desalination plants to be fully sustainable in the near future.

The Oak Ridge National Laboratory in the USA is exploring the development of the next generation nuclear power generation plants that may be a perfect fit for desalination plants. Instead of uranium, these plants use thorium and rubidium as a power source. A plant with capacity between 10 and 50 Megawatts will be suitable to power medium and large size desalination plants and will fit in the size of a typical street trash can. The key advantage of this energy source is that the nuclear fuel can be directly extracted in adequate quantities from the seawater desalination plant brine. Besides being readily extractible from the brine, a further advantage of these rare elements is that they cannot be used in building atomic weapons, thus providing desalination brine as the new fuel for peaceful use of atomic energy for the greater benefit of humanity. Therefore, seawater brine concentration and beneficial reuse holds the key to sustainable long-term desalination.

Despite of the few bumps on the road so far, sustainable and resilient water source management will embrace desalination in future, especially given the progress to date in: driving down the barriers for low energy desalination; extracting valuable resources from what was previously seen as waste streams, and increasing reliance on renewable energy sources.

\section{Acknowledgement}

None.

\section{Conflict of Interest}

No conflict of interest. 